\title{
Françoise CANON-ROGER
}

Université de Reims Champagne-Ardenne

f.canon-roger@orange.fr

\section{LES MOTS ENTRE TEXTES ET LANGUE : RÉFLEXIONS PRÉLIMINAIRES À L'ÉLABORATION D'UN LEXIQUE MULTILINGUE DES ARTS DE LA MARIONNETTE}

La définition $\mathrm{du}$ mot s'avère aussi élusive que celle du temps dont saint Augustin disait dans les Confessions (XIV) : "Qu'est-ce donc que le temps ? Si personne ne me le demande, je le sais; si je veux répondre à cette demande, je ne le sais pas. » Nous savons ce que sont les mots de manière intuitive, intime et affective même, mais toute définition plus scientifique se heurte à des difficultés que la plupart des linguistes ont bien montrées. A la définition on ne peut plus générale qu'en proposait Meillet : "Un mot résulte de l'association d'un sens donné à un ensemble de sons donné susceptible d'un emploi grammatical donné » (Meillet 1913/1 : 11), Marouzeau oppose qu'une telle définition a certes le mérite de "pouvoir s'appliquer à toute langue» mais qu'elle fait surtout ressortir «la difficulté de proposer une définition précise du mot» et il ajoute que "l'unité même du mot n'est pas aisément reconnaissable» (Marouzeau 1961 : 150). Jean Tournier, lexicologue de l'anglais, déclare que "la notion de mot n'a jamais pu être définie de façon pleinement satisfaisante » (Tournier $1991: 121$ ). Vendryes fait remarquer que la notion de mot gagne à rester vague dans la mesure où dans certaines langues comme le turc, le bantou ou le grœnlandais, elle recouvre une réalité bien différente de celle à laquelle nos langues nous habituent (Vendryes 1968 : 106). De même Martinet constate : «Il serait vain de chercher à définir plus précisément cette notion de mot en linguistique générale » (Martinet 1996 : 115) ; et Mounin de souligner la relativité de la notion : «il a été impossible de se mettre d'accord sur une définition du 
mot (car le mot est une unité très variable selon les langues)» (Mounin 1968 : 62). Mais c'est sans doute Saussure qui résume le mieux la difficulté et ses conséquences : "on s'est beaucoup disputé sur la nature du mot, et en y réfléchissant un peu, on voit que ce qu'on entend par là est incompatible avec notre notion d'unité concrète. (...) Il faut chercher l'unité concrète ailleurs que dans le mot. (...) La langue présente donc ce caractère étrange et frappant de ne pas offrir d'entités perceptibles de prime abord, sans douter pourtant qu'elles existent et que c'est leur jeu qui la constitue. C'est là sans doute un trait qui la distingue de toutes les autres institutions sémiologiques» (Saussure 1972 : 147-149).

\section{LES DIFFICULTÉS LIÉES À LA DÉFINITION DU MOT}

Les difficultés liées à la définition du mot existent aussi bien à l'oral qu'à l'écrit, en langue qu'en discours. En français, l'étymologie de mot rappelle ses liens avec l'oral mais pour signifier une déficience qui fait du mot un bruit. Il est issu du bas latin muttum 'son émis' de muttire' produire le son $m u^{\prime}$ d'où 'souffler mot, grommeler'. Il s'agit d'un terme de la langue parlée, rattaché à l'onomatopée $m u$ et en particulier à mutus 'son, bruit de voix qui n'a pas de signification', qui a donné aussi 'muet'. Dans les langues slaves, avec słowo par exemple, c'est la réception du son qui est retenue. Tandis que les familles germanique et romane emploient des mots dérivés des plus prestigieux verbum et parabola respectivement. Il n'est pourtant pas plus simple de définir ce qu'est un mot dans ces langues.

Toutes ces étymologies relient les mots avec l'oral. Or si certains phénomènes oraux ont justement pour fonction de permettre d'identifier les frontières des mots, d'autres ont pour effet de les brouiller. Ainsi dans certaines langues, la démarcation est assurée par l'accentuation ou liée à la position de certains groupes de phonèmes. Mais à l'inverse, les liaisons et l'agglutination rendent obscurs les contours des mots, ce dont témoignent en diachronie les phénomènes bien connus de déglutination ou segmentation erronée, comme en anglais celle qui a donné apron 'tablier' résultant du contact avec le déterminant : an aperon $>$ an apron selon un modèle établi en anglais.

Le critère graphique ne se révèle pas plus fiable d'un point de vue scientifique dans la plupart des langues, mais le latin présente des 
régularités remarquables qui ont permis l'émergence du mot graphique grâce aux moines copistes irlandais en particulier. Au IX siècle, les copistes commencent à décrocher les mots les uns des autres en tant qu'unités du discours, y compris les constituants grammaticaux, et introduisent des signes de ponctuation pour faciliter la lecture silencieuse. Mais la première étape de séparation en unités variant du syntagme à la phrase et disposées en lignes, dite per cola et commata ${ }^{1}$, fut la reprise par saint Jérôme de la méthode mise en œuvre dans des copies de Démosthène et de Cicéron (Manguel 1998 : 68-69). Il faut noter que ce découpage en unités reposait sur le sens, autrement dit les signifiants étaient construits et non pas donnés. En outre, ces unités de sens correspondent à des nécessités relevant de la rhétorique, à l'exemple des grands orateurs invoqués par Jérôme. Elles se distinguent en cela du découpage issu de la tradition logico-grammaticale qui considère le mot du point de vue des classes de mots ou parties du discours à l'intérieur de la phrase dont la définition est attribuée à Denys le Thrace dans sa Tekhnê Grammatikê : "la plus petite partie de l'énoncé construit portant un contenu de pensée». La tradition logico-grammaticale analyse la phrase comme l'application d'un prédicat à une entité, le premier étant un verbe et le second un nom (rhêma et ónoma). Au critère sémantique logique s'ajoute le critère morphosyntaxique de la possibilité pour le verbe de marquer l'expression du temps, ce qui n'est pas le cas pour le nom, du moins en grec. Les choses se compliquent cependant dès que l'on quitte les catégorèmes, dotés de sens, pour passer aux syncatégorèmes (súndesmos et árthron) qui sont censés ne pas en avoir et qui peuvent être composés de plusieurs éléments comme les conjonctions complexes. Les définitions du mot trouvent ici deux limites : il y aurait deux sortes de mots, les mots autonomes et les mots accessoires. Le sens résiderait dans les éléments lexicaux alors que les morphèmes grammaticaux sont vus comme des accidents qui les affectent. D'autre part, certaines unités peuvent être composées de plusieurs éléments, comme les conjonctions complexes (en anglais, par exemple, as soon as, in order to), ce qui nécessite une nouvelle distinction entre mots simples et mots composés. Cette manière

\footnotetext{
1 Prologue à la traduction du livre d'Ézéchiel : per cola scriptus et commata manifestiorem legentibu ssensum tribuit : "en lisant ce qui est écrit en cola et commata le sens se donne plus facilement. » Les portions et segments de texte sont disposés comme s'il s'agissait de vers.
} 
de définir le mot comme "un segment de discours compris entre deux espaces blancs» (Neveu 2004 : 198) est donc loin d'être universelle et fonctionnelle.

D'un point de vue morphologique, pour citer Vendryes : "La variété des procédés morphologiques fait que la définition du mot varie suivant les langues » (Vendryes $1968:$ 106). Le critère de la commutation en contexte identique devrait permettre de délimiter des unités fonctionnelles supérieures aux mots. Cette manipulation permet par exemple de montrer que si give up est susceptible de commuter avec abandon, on peut considérer qu'il s'agit d'un seul mot composé. Mais cette conclusion se trouve contredite lorsque l'on a recours au test de la séparabilité avec possibilité d'insertion sur l'axe syntagmatique : le cas de signifiants discontinus comme dans give up et sit in montre bien les limites de la procédure. S'il est possible d'insérer un pronom entre les deux éléments du composé, c'est que l'on a affaire à deux mots : Give it up ! Si cela n'est pas possible, comme dans sit in, il n'y en a qu'un. De fait, c'est la valence verbale qui fait la différence. Pourtant, le résultat obtenu par commutation est différent puisque sit est susceptible de commuter avec drive, ring ou cut et in avec back, down ou up.

Il semble que ce soit au niveau sémantique que la pertinence de l'unité 'mot' soit le plus souvent reconnue et depuis le plus longtemps. Aux deux catégories dégagées par les philosophes et grammairiens de l'Antiquité, celles des mots dénominatifs et des mots grammaticaux, les linguistiques de l'énonciation et de la pragmatique en ajoutent aujourd'hui une troisième, celle des indexicaux qui désignent en situation. Notons que ces mots-là ainsi que les mots grammaticaux sont désormais reconnus comme ayant un sens. Mais il faut aussi bien noter que le statut des mots dénominatifs, les lexèmes, ceux qui peuvent être dotés d'un référent, conservent en sémantique un statut privilégié tant il est vrai que la plupart des linguistiques contemporaines sont fondées sur la référence.

Il semble impossible de définir le mot à la racine. C'est ce que constatait Saussure : "Mais d'où prend-on d'abord qu'il y a un mot, lequel devra être considéré ensuite de différents points de vue ?». Et il poursuit en disant qu'en l'absence d'unité concrète, «éternellement donc le grammairien ou le linguiste nous donne pour entité concrète, et pour entité absolue servant de base à ses opérations, l'entité abstraite et relative qu'il vient d'inventer dans un chapitre précédent» (Saussure 2002 : 24). Autrement dit, le mot, unité préthéorique indéfinissable, sert néanmoins de 
base aux linguistes qui proposent d'autres appellations en fonction de leur spécialité et de l'objet qu'ils cherchent à construire. On dénombre ainsi, pour spécifier les appellations générales de mot simple, mot composé, vocable, en morphosyntaxe, synapsie, syntagme autonome, lexe et morphème qui inclut des unités inférieures au mot; en lexicologie, lexie (affixée, composée, complexe, prépositionnelle) ; en sémantique, des unités de signifié, sémème pour le morphème et sémie pour la lexie.

\section{L'ENVERS DE LA LIBERTÉ DES TERMES}

Compte tenu de toutes ces difficultés, on peut s'interroger sur la pertinence du concept de 'mot'. Si l'on continue à l'employer, c'est qu'on le confond souvent avec le signe et qu'une bonne partie de la linguistique repose sur la recherche d'unités qui soient données pour calculer le sens selon le principe de compositionnalité. Or, l'élaboration du sens procède du global vers le local ce qui veut dire que les 'unités' à retenir pour l'interprétation sont construites et non pas données et qu'elles peuvent ne pas correspondre aux mots. Mais si la difficulté à isoler les mots tient à la présence d'un contexte, il existe un domaine constitué justement d'unités isolées, celui de la terminologie, dont l'unité est le terme. La terminologie est l'un des domaines qui permet de poser le rapport entre mot et texte de manière particulièrement pertinente tout en offrant l'opportunité de développer une perspective critique sur ses a priori théoriques et ses pratiques.

Commençons par une définition du terme qui a le mérite de reposer sur celle du mot : "un terme est une unité signifiante constitué d'un mot (terme simple) ou de plusieurs mots (terme complexe), qui désigne une notion de façon univoque à l'intérieur d'un domaine. Ainsi, le terme s'oppose $\mathrm{au}(\mathrm{x}) \operatorname{mot}(\mathrm{s})$ par sa référence inhérente à un domaine » (Dubois et al. 1994). Avant de passer aux caractéristiques du terme, notons qu'à l'origine il appartient au domaine de la logique en syllogistique. Par exemple, dans Socrate est un homme, Socrate et homme sont des termes, c'est-à-dire des noms dont le sens est défini, délimité par leur référence. C'est ainsi que se poursuit la logique terministe (théorie de la dénotation) au Moyen Age jusqu'à la philosophie analytique et au positivisme logique dont procède la théorie de Wüster, le père de la terminologie (Rastier $1995: 2-3)$. 
Le terme possède un certain nombre de caractéristiques qui le différencient en particulier de la lexie. Un mot devient un terme par sa nominalisation pour les raisons indiquées ci-dessus ; par sa lemmatisation qui le débarrasse de ces accidents que sont les désinences, flexions et autres affixes pour conserver la substance du nom ou parfois du verbe mais le plus souvent nominalisé à l'infinitif. La fixité, l'univocité de sa référence, résulte de sa décontextualisation qui le met à l'abri des variations qui pourraient affecter ses occurrences. En outre, les termes n'ont pas de passé : la diachronie est exclue. Son seul contexte dans un réseau terminologique est constitué des autres termes auxquels il est lié dans les arborescences selon des relations qui structurent le lexique en langue et sont censées correspondre à des structures de pensée. Ensuite, le mot est érigé en type auquel correspond une définition qui énonce les conditions nécessaires et suffisantes - selon le positivisme logique de Carnap et Morris - pour que le terme soit pourvu de sa dénotation correcte. Un signifiant est mis en relation avec une notion-type qui désigne elle-même un objet selon le triangle sémiotique classique vox-conceptus-res dominant en linguistique encore aujourd'hui. Effectivement, le terme en tant que type est promu au statut de concept. En réalité, la boucle est bouclée puisque le concept est censé préexister au terme quand on a une vision instrumentale du langage, c'est-à-dire quand on pense que le langage habille la pensée. Côté res, les ontologies ont pour objectif la représentation des connaissances. De l'identité à soi de la chose découle celle du concept qui la représente et c'est ce lien qui assure l'univocité du concept. Selon cette conception héritée de la tradition logico-grammaticale, le concept est délié des langues. La recherche du concept est celle de l'invariant qui semble être l'objet de toute recherche alors que les objets empiriques se caractérisent par leur diversité, ce qui est beaucoup plus difficile à étudier. Cet aspect de la terminologie rejoint aussi une conception de la traduction identique à celle des tenants de la déverbalisation comme Jean-René Ladmiral qui appelle salto mortale le passage d'une langue à l'autre par les concepts qu'il croit communs à tous. Cette croyance dans l'universalité des concepts va de pair avec le désir de rédimer le défaut des langues en les soustrayant au temps et à l'espace. Déjà, et pour des raisons louables puisqu'il s'agissait de promouvoir la concorde entre les peuples, les caractéristiques universelles de Leibniz à Lodwick en passant par Wilkins, proposaient des ontologies, des inventaires des objets du monde, selon les genres, les 
différences et les espèces produisant des taxinomies. Mais comme Wilkins a été amené à le reconnaître, cette représentation abstraite et idéale ne convenait pas pour « définir les propriétés qui nous servent à reconnaître l'objet» (Eco 1994 : 291). Les propriétés liées à des circonstances particulières ont dû être exprimées en langue naturelle. Wilkins admettra finalement que le nom des choses ne suffit pas à nous instruire sur leur nature et que, pour ce faire, seules conviennent les discours en langues naturelles.

Le statut du terme et les a priori théoriques sur lesquels repose la terminologie amènent à s'interroger. Par exemple, le fait que le rapport du type à l'occurrence soit inversé pose question puisque, en principe, «les types ne préexistent pas aux occurrences, ils sont reconstruits à partir d'elles » (Rastier 1995 : 10). Les termes sont donc des artefacts. Ce renversement mérite d'être souligné : les types en langue ont une signification et les occurrences en discours un sens. Si l'on inverse cet ordre, «on place le sens sous la dépendance de la signification et le phénomène sous la dépendance de la norme » (Rastier : 11). Cela correspond au but normatif et prescriptif explicite de la terminologie tel qu'il est fixé par l'ISO'2. Plus radicalement, on est amené à se demander s'il existe vraiment des langues de spécialité qui seraient des sous-systèmes de la langue générale. C'est le postulat de départ pour la terminologie (Cabré 1998 : 115). C'est aussi ce que disait Coseriu à propos du lexique : «il y a un lexique structuré linguistique, et un lexique 'nomenclateur' et terminologique». Et il proposait de rejeter la terminologie dans la linguistique externe (Coseriu 2001 : 224-225). Mais il affirmait aussi : «Il est vain, par conséquent de vouloir interpréter les structurations linguistiques du point de vue des prétendues structures de la réalité : il faut commencer par constater que ce ne sont pas des structures de la réalité mais des structures imposées à la réalité par l'interprétation humaine (...). » (Coseriu : 227-228). La distinction entre lexique général et lexique spécialisé n'a pas lieu d'être parce qu'il n'y a pas de lexique général : «tout usage linguistique est situé dans et par une pratique déterminée» (Rastier $1994: 78$ ). Autrement dit, tout texte, oral ou écrit, relève d'un discours entendu cette fois comme pratique sociale (juridique, médicale, technique, littéraire, etc.) et d'un genre particulier. Les textes techniques ne diffèrent donc pas des autres textes

2 ISO : International Organization for Standardization ; normes internationales couvrant presque tous les secteurs du monde économique, de l'industrie et de la technologie. 
relevant d'autres discours qui comportent également des caractéristiques linguistiques propres (Rastier 2011 : 71-98).

La terminologie qui débouche sur des ontologies vise des représentations de connaissances découplées des langues. Les concepts mis en œuvre par la terminologie, ne sont que des sémies (contenu de lexie) stabilisées par les normes d'une discipline et séparées de leur contexte. Ce que les terminologues appellent « concept est le signifié d'un mot dont on décide de négliger la dimension linguistique» (Rastier 1995 : 11). Or cette approche purement paradigmatique est insuffisante car rien ne peut être représenté en langue qui n'ait auparavant été décrit en contexte (Rastier 1994 : 62). Le but d'une entreprise lexicologique étant de faciliter la production, l'interprétation et la traduction de textes, celle-ci a tout intérêt à être conduite à partir des textes pour y revenir. Dans les 'domaines de spécialité, dont on a vu qu'ils relèvent en réalité de discours propres à une pratique sociale parmi d'autres, cette abstraction ne peut pas être considérée comme suffisante pour une description du domaine en question car l'approche peut varier en fonction des discours, des genres et des contextes.

\section{UNE APPROCHE CONTEXTUELLE DU LEXIQUE}

Avant d'en venir à cette approche contextuelle du lexique et à ses conséquences méthodologiques, il importe de considérer les spécificités du discours dans lequel se trouvent les mots à étudier. Ainsi pour élaborer un lexique multilingue des arts de la marionnettes ${ }^{3}$, la première étape consiste à délimiter un corpus de textes lié à cette pratique sociale artistique. Or les arts du spectacle donnent lieu à des textes qui relèvent de discours différents. On entend par discours « des types d'usages linguistiques codifiés qui correspondent à des pratiques sociales différenciées et articulent des domaines sémantiques propres»(Rastier 2001 : 226). L'intitulé «les arts de la marionnette » correspond à un thème qui peut être traité selon le discours littéraire lorsque Kleist, Charles Nodier ou

3 «Les mots de la marionnette» : projet de l'Institut International de la Marionnette de Charleville-Mézières auquel prend part le Centre Interdisciplinaire de Recherches sur les Langues et la Pensée (CIRLEP EA 4299) et en particulier le laboratoire "Intercompréhension et didactique du plurilinguisme». Ce projet de recherche en est à son stade initial. 
Georges Sand s'en emparent. Cette dernière pratique même différents genres qui ont en commun ce thème. Ainsi «Le théâtre des marionnettes de Nohant » peut être classé parmi les textes autobiographiques tandis que l'Homme de neige (1859) dédié par George à son fils Maurice Sand est un roman qui s'inspire de leurs séances de montreurs de marionnettes à Nohant. Mais les arts de la marionnette font aussi l'objet de textes techniques, pédagogiques, académiques, juridiques, journalistiques, publicitaires et sans doute d'autres encore. Un lexique qui ne retiendrait que les textes les plus techniques passerait à côté du but de cette recherche. De plus, pour ce type de pratique, les dimensions diachronique et diatopique ne sauraient être négligées. Loin d'être donné, le corpus doit donc être soigneusement délimité en fonction de sa pertinence pour la tâche à accomplir. Il n'est pas exclu de devoir établir des sous-corpus compte tenu de cette multiplicité de discours et des genres qui les manifestent. Ainsi par exemple, du discours juridique relèvent les arrêtés qu'ils soient d'autorisation des spectacles, de réglementation ou d'interdiction et de censure, les ordonnances, les textes relatifs aux droits d'auteur etc. Mais dans la mesure où le lexique à construire est destiné aux professionnels de la marionnette eux-mêmes, ce discours pourra ne pas être prioritaire. Il peut néanmoins fournir des éléments de classification complémentaires. Par exemple, dans les textes, l'animation des marionnettes géantes est apparentée au mime et fait partie des spectacles des 'arts de la rue' qui bénéficient de droits d'auteur sur la chorégraphie/déambulation contrairement à d'autres types de spectacles. Dans la perspective de l'élaboration d'un lexique multilingue, le genre est d'une importance capitale. C'est une équivalence de genre qui peut fournir le pivot de la traduction. En l'absence d'identification possible entre une langue et une autre, c'est-à-dire entre systèmes linguistiques différents, il faut trouver un élément qui donne lieu à une production linguistique mais qui n'appartienne pas strictement au système de la langue. Ce qui rend la traduction et l'intercompréhension possible, c'est l'analogie relative des pratiques sociales et donc celle des discours et des genres qui leur sont liés.

L'extraction des mots qui formeront le lexique résulte de la construction des taxèmes c'est-à-dire de classes sémantiques minimales définies en fonction des situations d'usage. Le taxème est une classe lexicale en langue parce que les unités qui y sont indexées ont toutes au moins un sème générique inhérent commun. Mais la sémantique des textes, parce 
qu'elle a précisément pour objet les textes, ne 'paradigmatise' qu'en fonction de ce qui apparaît sur l'axe syntagmatique. Le mot ou sémème se trouve indexé dans un taxème qui relève de la langue mais il est constitué à partir d'un texte ou d'une situation concrète c'est-à-dire en discours. Il ne s'agit pas de termes au sens décrit plus haut mais de mots dans les textes grâce à une approche textuelle du lexique. Les 'mots' ou plus précisément les sémèmes inclus dans un taxème forment un ensemble dont on peut dire qu'il se situe entre langue et texte. L'approche est bien orientée du global vers le local, du type de discours aux sémies. Les classes lexicales varient avec les pratiques sociales et il est douteux que le lexique soit structuré en langue. Un champ peut se composer de taxèmes relevant de domaines différents ${ }^{4}$, en particulier de différents domaines techniques. Notons que les taxèmes ainsi définis évoluent vite : 'robot' est inclus depuis peu dans le taxème des types de marionnettes. Le taxème n'est pas figé comme peut l'être une ontologie mais il n'est pas non plus sans relations comme l'est une liste. Ces relations sont uniquement sémantiques. Les taxèmes se structurent entre eux pour former des champs sémantiques. Enfin, les dimensions sont des classes de très grande généralité qui se manifestent selon des oppositions : /végétal/ vs /animal/vs /humain/ ou encore /animé/vs /inanimé/, qui peuvent être particulièrement pertinentes dans le cadre d'une étude des mots de la marionnette. Les dimensions peuvent diviser les domaines : dans le domaine //marionnettes//, le taxème des manipulateurs /animé/ est distinct de celui des poupées /inanimé/ mais comme il s'agit précisément d'animer ces poupées, ce trait deviendra essentiel dans la distinction entre les gestes /animé/ de l'agent et le mouvement du patient qui est un simulacre de corps /inanimé/. Là encore l'apparition de 'robot' dans l'intitulé d'une journée d'étude active cette opposition : "Marionnettes, automates et robots : miroirs de $1^{\prime}$ Homme ? $»^{5}$. C'est à partir du relevé des syntagmes équatifs, une énumération par exemple comme ici, que s'élabore l'inventaire des taxèmes. Dans une démarche orientée du global vers le local, le but est de parvenir aux classes les plus restreintes où l'on peut faire ressortir les sèmes spécifiques qui distinguent leurs éléments et structurent ces taxèmes. Tout l'intérêt de ces constructions tient à la mise en évidence des sèmes spécifiques particulièrement pertinents pour

4 Ils figurent dans les dictionnaires en tant qu'indicateurs lexicographiques.

5 Journée d'étude organisée par le Musée de Gadagne (Lyon) le 12 octobre 2013. 
l'usage et la traduction. Dans un lexique spécialisé, qui est en principe destiné aux experts, il est peu probable que les éléments les plus génériques soient dignes d'intérêt.

Bien qu'il ne s'agisse pas d'interpréter les textes du corpus mais d'élaborer un lexique, certains morphèmes grammaticaux seront inclus puisque les sèmes ignorent les classes de mots et que parmi les éléments lexicaux il conviendra de tenir compte des lexies complexes. Ainsi il faudra retenir non seulement les noms mais aussi les verbes et les lexies complexes relevant de collocations et de la phraséologie. Les types de marionnettes traditionnelles sont distingués par le groupe prépositionnel qui modifie le nom 'marionnette' : //marionnette//à gaine/à tringle/à fils/ à baguettes/à clavier/à tige. Chaque groupe prépositionnel définit un mode de manipulation : /par en-dessous/, /par le dessus/haut/, etc. Mais sur le même modèle syntaxique, on trouve aussi 'marionnette à transformation' correspondant à un type de marionnette qui change d'apparence ou qui cache des figurines ou d'autres marionnettes dans son costume. C'est le cas de Gigogne, mère de Polichinelle, qui laisse soudain apparaître ses 'enfants' sous ses jupes. De là l'expression entrée dans l'usage populaire avoir un polichinelle dans le tiroir. Il apparaît clairement qu'à une conception taxonomique particulièrement inadaptée à ce domaine, on préfèrera une approche syntagmatique qui relève les déterminations que le terme reçoit des textes d'où il est issu. Dans l'exemple proposé ci-dessus, la différence de sens entre les deux constructions GN à GN tient à la différence entre leurs schémas actanciels respectifs. En outre, seule la dimension textuelle peut permettre de mettre en évidence les inégalités qualitatives des termes entre eux. Ce traitement qualitatif du lexique n'est pas spontanément associé au discours technique que l'on se représente comme neutre et objectif. Or il importe de pouvoir rendre compte de différences dans l'emploi de termes proches. Ainsi le terme que l'on trouve pour signifier 'technique pour relier la marionnette à son contrôle par des fils' est ensecret ou ensecrètement. C'est une étape cruciale dans la fabrication de la marionnette qui doit aboutir à un équilibre parfait. L'action est donc valorisée puisqu'elle relève du secret de fabrication assimilable à celui des instruments de musique ou des pigments et vernis en peinture : « Le marionnettiste amoureux de son métier ne se lasse pas de perfectionner l'instrument dont il se sert et de se créer une technique bien à lui, laborieusement mise au point - 'l'ensecret', comme on dit dans la corporation - qu'il garde jalousement et qu'au sur- 
plus les autres professionnels, ses rivaux, se font un point d'honneur de respecter» (Baty 1972 : 9). On note la même intensité liée au commentaire métalinguistique chaque fois que le mot ou ses dérivés (ensecréter, désensecréter) sont employés : "Mais il est bien entendu que chaque marionnettiste a ses inventions. Et ce n'est pas seulement pour créer l'illusion que la plupart des marionnettes à fils ne sont pas manipulées à vue ; c'est pour entretenir le secret. L'ensecrètement est d'ailleurs aussi le mot qui a longtemps désigné le 'contrôle' lui-même.» (Fournel 1982 : 88). Mais une fois l'opération nommée, on trouve des équivalents comme mettre sur fils, accrocher/poser/fixer les fils. L'intensité qualitative conférée à ce mot est propre au français puisqu'on trouve stringing en anglais et encordelar en espagnol, deux termes transparents. De même 'la pièce de bois sur laquelle sont fixés les fils' est lexicalisée de plusieurs façons différentes dont l'usage n'est pas indifférencié : contrôle, attelle, airplane, croix. Il faudrait étudier leur distribution et leur fréquence selon le genre des textes dans lesquels ils apparaissent mais on peut faire l'hypothèse que dans cette série le premier des termes est plus neutre qualitativement que le dernier.

Compte tenu de la multiplicité des objectifs affichés, il sera sans doute nécessaire de prévoir plusieurs volets à ces recherches mais il ne fait pas de doute que compte tenu de l'intérêt linguistique, culturel et anthropologique du projet, le cadre théorique présenté ici est le plus adapté. Face au postulat ontologique, aux puissants universaux, invariants, primitives, mieux vaut «l'humilité du sème » (Valette $2008: 45$ ) dont la dépendance à la langue, à la pratique sociale et à la culture est déterminante.

\section{RÉFÉRENCES}

Baratin M., Desbordes F., 1981, L'analyse linguistique dans l'antiquité classique, Paris, Klincksieck.

Baty G., Chavance R., 1972, Histoire des marionnettes, PUF, Que sais-je ?

Briand M., 2014, Les arts de la marionnette : un patrimoine multiple à définir, à conserver et à mettre en valeur, Mémoire de M2, direction Julien Schuh, Université de Reims Champagne-Ardenne.

Cabré M.-T., 1998, La terminologie. Théorie, méthode, application, Paris, Armand Colin.

Canon-Roger F., Chollier C., 2008, Des genres aux textes. Essais de sémantique interprétative en littérature de langue anglaise, Arras, Artois Presses Université.

Coseriu E., 2001 [1966], «Vers l'étude des structures lexicales », in : L'homme et son langage, Louvain, Peeters, 215-252.

Dubois J. \& al., 1973, Dictionnaire de linguistique et des sciences du langage, Larousse. 
Eco U., 1994, La recherche de la langue parfaite, Paris, Seuil.

Fournel P. (éd.), 1982, Les marionnettes, Paris, Bordas.

Habert B., Nazarenko A., Salem A., 1997, Les linguistiques de corpus, Paris, Armand Colin.

Lorentz N., 2011, Au corps des choses. Étude sur la marionnette contemporaine occidentale, Master 2, direction Frédéric Pouillade, Université de Paris-Sorbonne.

Manguel A., 1998, Une histoire de la lecture, Paris, Actes Sud.

Marouzeau J., 1961, Lexique de la terminologie linguistique, Paris, Paul Geuthner.

Martinet A., 1996, Éléments de linguistique générale, Paris, Armand Colin.

Meillet A., 1913/1, Revue de métaphysique et de morale, Paris, Armand Colin.

Mounin G., 1968, Clefs pour la linguistique, Paris, Seghers.

Neveu F., 2004, Dictionnaire des sciences du langage, Paris, Armand Colin.

Rastier F., Cavazza M., Abeillé A., 1994, Sémantique pour l'analyse. De la linguistique à l'informatique, Paris, Masson.

Rastier F., 1996, «Le terme : entre ontologie et linguistique », Texto ! [en ligne], disponible sur : http://www.revue-texto.net/Inedits/Rastier/Rastier_Terme .html.

Rastier F., 2001, Arts et sciences du texte, Paris, PUF.

Rastier F., Cavazza M., Abeillé A., 2002, Semantics for Descriptions. From Linguistics to Computer Science, translated by R. Lawrence Marks, Stanford, California, Center for the Study of Language and Information.

Rastier F., 2004, «Ontologie(s) », Texto ! [en ligne], disponible sur : http://www. revue-texto.net/Inedits/Rastier/Rastier_Ontologies.html.

Rastier F., 2009, Sémantique interprétative, Paris, PUF.

Rastier F., 2011, La mesure et le grain. Sémantique de corpus, Paris, Honoré Champion.

Saussure F. de, 1972, Cours de linguistique générale, Paris, Payot.

Saussure F. de, 2002, Écrits de linguistique générale, Paris, Gallimard.

Slodzian M., 2007, « Rationalisation des langues et terminologie : d'Ogden à Catford », in : Hermès, La Revue, 2007/3 n 49, 59-68. URL : www.cairn.info/ revue-hermes-la-revue-2007-3-page-59.htm.

Tournier J., 1991, Structures lexicales de l'anglais, Paris, Nathan.

Valette M., 2010, "Approche textuelle du lexique», TEXTO !, [en ligne], Volume XV n ${ }^{\circ}$ 2. Coordonné par Carine Duteil-Mougel. URL : http://www. revue-texto.net/index.php?id=2613.

Valette M. (éd.), 2008, «Textes, documents numériques, corpus. Pour une science des textes instrumentés », Syntaxe et sémantique 9, Presses Universitaires de Caen.

Vendryès J., 1968, Le Langage : Introduction linguistique à l'histoire, Paris, Albin Michel.

Williams G. (éd.), 2005, La linguistique de corpus, Presses Universitaires de Rennes. 


\section{LES MOTS ENTRE TEXTES ET LANGUE : RÉFLEXIONS PRÉLIMINAIRES À L'ÉLABORATION D'UN LEXIQUE MULTILINGUE DES ARTS DE LA MARIONNETTE}

\section{Résumé}

Une fois précisées les difficultés qui s'attachent à la définition du mot comme unité linguistique, on pourra aborder les relations que celui-ci entretient avec le système d'une langue d'une part et avec les textes d'autre part. La terminologie est l'un des domaines qui permet de poser ce rapport de manière particulièrement pertinente tout en offrant l'opportunité de développer une perspective critique sur ses a priori théoriques et ses pratiques. Ces réflexions proposent une présentation, à un stade initial, de travaux de recherches en sémantique des textes qui conduiront à l'élaboration d'un lexique multilingue des arts de la marionnettes. Les mots érigés en termes ne font pas la langue puisque seuls sont retenus en général les noms et les verbes. Or dans une perspective descriptive, même appliquée à un domaine particulier, il est souhaitable d'inclure d'autres parties du discours, d'élargir le relevé et d'y inclure les lexies complexes. Il importe également de fonder le choix d'un terme sur la prise en compte de ses diverses lexicalisations et des variations qui affectent ses occurrences en contexte. A l'inverse, un texte n'est pas fait de mots, sauf à le concevoir, comme c'est souvent le cas à l'ère du numérique, comme 'un sac de mots' à traiter de manière quantitative. Il importe au contraire de tenir compte des paliers de complexité et des inégalités qualitatives qui caractérisent les textes, particulièrement en fonction du discours et du genre auxquels ils appartiennent. Dans cette perspective théorique, les classes sémantiques élaborées en contexte participent à la fois des textes et des langues comme systèmes.

Mots-clés : sémantique des textes, lexicologie, terminologie, texte, genre, contexte, variation, marionnettes

\section{WORDS BETWEEN TEXTS AND LANGUAGE: PRELIMINARY REFLECTIONS ON BUILDING A MULTILINGUAL LEXICON OF PUPPETRY}

\section{Summary}

Many linguists have emphasised the difficulties in attempting to define what a word is. The notion differs according to the language under study and it even proves irrelevant for a certain number of languages. Although 'word' has not quite ceased to be used for convenience with a fuzzy extension, it is replaced by metalinguistic terms coined to fit the units specific to lexicology, semantics or terminology. There remains the central question of the nature and boundaries of these units and of their relation with a linguistic system, on the one hand, 
and with the texts in which they occur, on the other. Consideration given to the theoretical framework of terminology leads to a critical viewpoint on practices that rest on logical principles incompatible with a context sensitive approach to lexical semantics even in a technical field such as the art of puppetry dealt with here. A language for specific purposes is not a linguistic sub-system since any text, oral or written, originates in a particular social situation manifested by its discourse and genre. Texts are not strings of words. Text semantics provides a textual approach to the lexicon in which the entries in a semantic class are selected contextually thus partaking of both texts and language as a system.

Key words: interpretative semantics, lexicology, terminology, text, genre, context, variation, puppetry 